\title{
Why Mathematics Is Universal Multidisciplinary Science
}

\author{
Sergey Krylov \\ Department of Computer Science, Samara State Technical University, Russia
}

Copyright (C) 2015 by authors, all rights reserved. Authors agree that this article remains permanently open access under the terms of the Creative Commons Attribution License 4.0 International License

\begin{abstract}
The paper shows meta-mathematical prerequisites for basic concepts of rigorous science called mathematics. These concepts explore a very simple idea concerning the hypothesis that all surrounding physical processes are basically algorithmic processes - as understandable as well as partially or fully incomprehensible ones. Mathematics is very successful in studying, formal describing and utilizing of such processes, because mathematics is based on similar algorithmic ideas, methods, and structures. These facts allow us to formulate more precisely useful mathematical (meta-scientific) concepts concerning some well-known scientific problems in various rigorous theories, including the theory of "object calculus", the theory of automatic cognition, the theory of biological evolution, the theory of heterogeneous electronic systems, the theory of logics in various chemical transformations, the basic architecture of completely programmable universal (multi-purpose) synthesizers-analyzers for various objects, and so on.
\end{abstract}

Keywords Algorithms, Physical Objects, General System Theory, General Formal Technology, Object Properties, Object Functionalities, "Object Calculus", Meta-science

\section{Introduction}

Idea to use fundamental algorithmic structures to explain the furniture of our world has a very long history - as well as Metaphysics itself. Aristotle, the creator of Metaphysics, wrote: "...some things are produced out of each other by combination, others by separation..." [1]. There were no notion of "algorithm" in that times, but we can see in Aristotle's words an attempt to emphasize the importance of basic algorithmic operations such as synthesis ("combination") and decomposition ("separation") of various objects. Moreover, next in his ninth chapter of Metaphysics, Aristotle tried to connect these ideas with the notion of number.

More precisely a similar idea was expressed by Augusta Ada Lovelace in 1843 in her "Notes by the Translator", devoted to the first mechanical computer that was invented by Charles Babbage. Explaining the essence of term "operation" she wrote: "...by the word operation, we mean any process which alters the mutual relation of two or more things, be this relation of what kind it may. This is the most general definition, and would include all subjects in the universe... But the science of operations, as derived from mathematics more especially, is a science of itself, and has its own abstract truth and value; just as logic has its own peculiar trust and value, independently of the subjects to which we may apply its reasoning and processes..." [2].

In the recent years the similar ideas were expressed by many authors. For example, in 1983 B. Jones in his paper devoted to Algorithms Theory and its importance for General System Theory (GST) emphasised: "Algorithms exist in many different disciplines. Here, our goal is to find isomorphic models and lows or principles in different fields..." [3].

Fontana and Buss in 1996 wrote: "But if the objects become the variables of the system, we would need a 'calculus of objects' like we have a differential calculus for numerical values" [4]. Moreover, in this paper they emphasised: "In Nature, interaction involves objects directly and never by a numerical value describing them..."

In $2000 \mathrm{~J}$. Schmidhuber published his work called 'Algorithmic Theories of Everything', in which he described any object $X$ by a string of symbols (bits). He defined $X$ as follows: "..$X$ should be representable by a possibly infinite bit string $x$ such that there is a finite, possibly never halting program $p$ that computes $x$ and nothing but $x$ in a way that modifies each output bit at most finitely many times; that is, each finite beginning of $x$ eventually converges and ceases to change" [5]. That is, at every moment of time each object $X$ is represented by finite constant substring of possibly infinite string. Unfortunately, he has achieved only ordinary results.

In $2014 \mathrm{M}$. Tegmark have suggested an opposite idea that outer world is constructed on the basis of mathematics [6]. In spite of the seeming absurdity, this idea has some signs of truth, especially if we expand a central mathematical concept called "algorithm" to the wider set of objects including not only numbers and symbols, but also real things.

\section{General Formal Technology and Mathematics}


In 1986 the paper called 'Formal Technology and Universal Systems I, II' was published in Russian Journal "Kibernetika" (at present the Journal is called "Cybernetics and System Analysis") [7]. It is necessary to note, that in Russian the word "technology" has a more general meaning than in English. In Russian term "technology" means "the set of operands (i.e. the objects of operations), the set of operations, and the sequences of such operations on operands (i.e. algorithms containing precise sequences of operations with specific operands)". That term "technology" in Russian means "the algorithm or some set of algorithms defining processes of production specific materials or things (objects)". Hereinafter, referred to the word "technology", we should consider it in such "Russian" sense.

In this paper some elements of algorithmic theory over physical objects were introduced. This theory, called "General Formal Technology" (GFT or "Formal Technology" for short), describes any algorithmic system $\mathbf{T}$ over physical objects as a triplet:

$$
\mathbf{T}=<\mathbf{B}, \mathbf{F}_{\mathbf{T}}, \mathbf{F}_{\mathbf{A}}>
$$

where $\mathbf{B}$ is a set of real physical objects (or abstract ones, i.e. models of physical objects) obtained from other objects of $\mathbf{B}$ or (and) some objects of finite set A. Objects of $\mathbf{A}$ are called basic elements, and $\mathbf{A} \subseteq \mathbf{B}$. Therefore the most compact representation of any formal technology $\mathbf{T}$ is the following triplet:

$$
\mathbf{T}=<\mathbf{A}, \mathbf{F}_{\mathbf{T}}, \mathbf{F}_{\mathbf{A}}>.
$$

Objects of $\mathbf{B}$ are called constructions and, as mentioned above, they are obtained from basic elements and (or) some constructions of $\mathbf{B}$ by means of finite set of technological operations $\mathbf{F}_{\mathbf{T}}$ and a finite set of operations of analysis $\mathbf{F}_{\mathbf{A}}$. The terms "object", "element", and "construction" are equivalent in GFT frameworks. The symbol $\mathbf{F}_{\mathbf{T}}$ in (1) and (2) denotes a finite set of finite-place "technological operations", and the finite set $\mathbf{F}_{\mathbf{A}}$ is a finite set of finite-place "operations of analysis" with objects of $\mathbf{B}$.

Any physical object $x \in \mathbf{B}$ (or $x \in \mathbf{A}$ ) in modern GFT is described very similar to a description of any program essence ("object") in Object Oriented Programming (OOP), i.e. as a following pair:

$$
x=<\Gamma_{x}, \mathbf{M}_{x}>,
$$

where $\Gamma_{\boldsymbol{x}}=\left\{\gamma_{x l}, \gamma_{x_{2}}, \ldots, \gamma_{x n}\right\}$ is a finite set of physical properties of object $x$, and $\mathbf{M}_{\boldsymbol{x}}=\left\{\gamma_{x j}=\varphi_{x j}\left(\gamma_{s k}, \gamma_{x l}, \ldots, \gamma_{q m}\right) ; \ldots\right\}$ is a finite set of functionalities of object $x$, i.e. a set of functions, defining the exact values of one $\left(\gamma_{x j}\right)$ or/and many physical properties of object $x$ by means of other properties (for example, $\left.\gamma_{x l}\right)$ of the same object $x$ or by properties $\left(\gamma_{s k}, \ldots, \gamma_{q m}\right)$ of other objects $(s, \ldots, q)$, which interact with properties of object $x$. In OOP sets like $\mathbf{M}_{x}$ are often called "methods". In canonical GFT frameworks set $\mathbf{M}_{x}$ has no functionalities at all [7]. In modern versions of GFT set $\mathbf{M}_{x}$ can have any finite number of functions (functionalities) like $\varphi_{x j}$ - as many as it is necessary for actual representation of $x[8,10]$. An object $x$ can be denoted also as $O_{x}$ or $O_{i}$ when representation (3) is very important - see, for instance, $[8-10]$.
At the same time a main algebraic structure $\mathbf{A}$ in mathematics has, according to Malcev, the following formal representation:

$$
\mathrm{A}=<\boldsymbol{A}, \mathbf{\Omega}_{F}, \mathbf{\Omega}_{P}>,
$$

where $\boldsymbol{A}$ is a base set of elements in $\mathbf{A} ; \boldsymbol{\Omega}_{F}$ is a set of (mathematical) operations; and $\boldsymbol{\Omega}_{P}$ is a set of predicates on $\boldsymbol{A}$ [11].

It is clear from representations (1) and (4) that we can define any algebraic system $\mathbf{A}$ as a kind of formal technology $\mathbf{T}_{\mathbf{M}}$ :

$$
\mathbf{T}_{\mathbf{M}}=<\boldsymbol{A}=\mathbf{B}, \boldsymbol{\Omega}_{F}=\mathbf{F}_{\mathrm{T}}, \boldsymbol{\Omega}_{\boldsymbol{P}}=\mathbf{F}_{\mathrm{A}}>.
$$

The essences of definitions (1) and (4) are very similar from the formal point of view. The differences are as follows:

1. The set $\mathbf{B}$ involves physical and abstract objects (as abstract models of physical objects), while $\boldsymbol{A}$ involves only abstract (mathematical) ones.

2. The set $\boldsymbol{\Omega}_{\boldsymbol{F}}$ involves only a finite set of mathematical operations, while $\mathbf{F}_{\mathbf{T}}$ involves not only mathematical operations, but also a finite set of real (or imaginable) technological operations including operations of object syntheses and object decompositions [7].

3. The set $\boldsymbol{\Omega}_{P}$ involves only a finite set of mathematical predicates, while $\mathbf{F}_{\mathbf{A}}$ involves a finite set of "analytical" operations that "measure" various physical properties of objects $x$ :

$$
P_{j}(x) \rightarrow r_{x},
$$

where $P_{j} \in \mathbf{F}_{\mathbf{A}}, r_{x} \in \mathbf{R}_{x}$, and $\mathbf{R}_{x}$ is a finite set of round-off natural or rational numbers necessary for practical application of values $\gamma_{x j}$, which are important for the used model of $x ; \gamma_{x j} \in \Gamma_{x}[8,10]$. One can easily prove that if an operation of analysis $F_{i} \in \mathbf{F}_{A}$ has only a finite number of results belonging to the finite subset of rational numbers $\mathrm{R}$, then all of these results can be represented by a finite number of proper predicates [12]. So, in fact, representations (6) and (2) are very close to the essence of representation (4).

4. Formal representation of finite abstract mathematical objects differs from that of physical ones. Indeed, if we use notation (3) to represent any finite abstract mathematical object $x$, then we should write the following:

$$
x=<\gamma_{x}>,\left(\text { or } x=<\left\{\gamma_{x 1}, \gamma_{x 2}, \ldots, \gamma_{x n}\right\}>\right)
$$

where $\gamma_{x}\left(\gamma_{x 1}, \gamma_{x 2}, \ldots, \gamma_{x n} ; n \in \mathrm{N}\right)$ is the main property (properties) of finite abstract mathematical object $x$ representing quantity, because each finite string of symbols describing any finite abstract quality of object $x$ can be transformed into proper finite natural number and vice versa (see, for example, Gödel's numbering and Gödel's $\beta$ function [13] or complete proof of this conclusion in [9]).

Equations (3) and (7) could explain, first of all, the following facts: (i) why Schmidhuber's concepts do not work properly; and (ii) why it is so difficult to construct a pure mathematical theory of evolution $[10,14,15]$. Indeed, 
equation (7) shows that pure mathematical objects $x$ have not contained any physical properties at all (Aristotle), therefore it is very difficult to save complete analogies among physical world and its pure mathematical representation (resembles Schmidhuber's concepts), if we do not take into account the essence of equation (3). The same principles are true for Chaitin's attempts to construct the Theory of Evolution on the basis of Algorithm Complexity Theory [14].

\section{Properties of Formal Technological Systems}

One of the most interesting items in these fields is the mathematical explanation of life origins. Indeed, Gregory Chaitin wrote: “...if you could do that, it might shed some light on how general the phenomenon of evolution is, and whether there's likely to be life elsewhere in the universe. Of course, even if mathematicians never come up with such a theory, we'll probably find out by visiting other places and seeing if there's life there... But anyway, von Neumann had proposed this as an interesting question, and at one point in my deluded youth I thought that maybe program-size complexity had something to do with evolution... But I don't think so anymore, because I was never able to get anywhere with this idea..." [14]. If we take into account equation (3) to represent a functionality $\boldsymbol{\Phi}$ of any linear molecular construction $y$ containing $n$ different molecules $x_{i}$ (with their physical properties and functionalities), then it immediately becomes clear that the total functionality $\boldsymbol{\Phi}$ of $y$ depends on the number $n$ of molecules $x_{i}$ in accordance with the following estimation:

$$
\Phi(y) \approx n \log _{2} s,
$$

where $s$ is a physical parameter defining an average value of functional states per one position of object (molecule) $x_{i}$ in a linear construction $y$ for the given technology $\mathbf{T}$. If $\mathbf{T}$ does not contain functional objects at all, i.e. $s=1$ for every $x_{i}$ (as it occurs in mathematics), then

$$
\boldsymbol{\Phi}(y)=n \log _{2} 1=0 \text { for any } n .
$$

In the case (8), object-molecules have many functional states $(s>1), \log _{2} s>0$, and the total functionality $\boldsymbol{\Phi}(y)$ will increase with the length of $y$, therefore it can achieve the level of self-reproduction. For example, in von Neumann self-reproducing cellular automata, $s=29$ [16].

On the other hand, equation (3) allows us to reformulate some mathematical points of view concerning data processing systems. Indeed, we prefer to describe and develop such systems as homogeneous ones for the following reasons: i) the complete description of the whole homogeneous system is easier for digital, as well as for analog types of data; ii) various transformations of different data use easier mathematical formulas and technical realizations in the case of homogeneous types of data; iii) it is much easier to use homogeneous mathematical methods to optimize and to test designed electronic circuits (systems).
But it is not true for the Nature! Nature prefers to use for the same purposes heterogeneous "gates", "amplifiers", and "signals" [17-19]. These heterogeneous approaches make appropriate devices more compact, more suitable and self-synchronized!

In accordance with (3) we describe, for instance, properties and functionalities of a two-input logic gate "nand" $\mathrm{LG}_{x}$ as follows:

$$
\mathrm{LG}_{x}=\left\langle\boldsymbol{\Gamma}_{\boldsymbol{x}} ; \mathbf{M}_{x}>=\left\langle\left\{\gamma_{1}, \gamma_{2}, \gamma_{3}\right\} ;\left\{\gamma_{3}=\right\rceil\left(\gamma_{1} \& \gamma_{2}\right)\right\}>,(10)\right.
$$

where $\gamma_{1}, \gamma_{2}, \gamma_{3} \in\{0,1\}$ are seen as parameters, $\gamma_{1}, \gamma_{2},-$ are input binary data (input parameters); $\gamma_{3}$ - output binary data (output parameter); $\mathbf{M}_{x}-$ describes a logical function "not-and" ("nand") for $\gamma_{3}$. This logical function does not depend on signal types - be these binary signals potentials, currents, frequencies or even capacity or resistive levels and their combinations $[17,18]$. These circumstances allow us to use for a designing of various heterogeneous digital or analog systems a wider range of functional blocks than it is possible for homogeneous systems. Sometimes it leads to unusual circuits.

The very similar situation has taken place in chemical reactions (transformations). For example, suppose we have a following reaction among chemicals $a$ and $b$ with properties $\Gamma_{a}$ and $\Gamma_{b}$ respectively to obtain a new chemical $c$ with properties $\Gamma_{c}$ :

$$
a+b \rightarrow c=<\Gamma_{a}, \mathbf{M}_{a}>+<\Gamma_{b}, \mathbf{M}_{b}>\rightarrow<\Gamma_{c}, \mathbf{M}_{c}>,
$$

where properties $\Gamma_{a}, \Gamma_{b}$ and functionalities $\mathbf{M}_{a}, \mathbf{M}_{b}$ are intended for a rapprochement of molecules $a$ and $b$ and to transform them into molecule $c$ with a new set of properties $\Gamma_{c}$ and functionalities $\mathbf{M}_{c}$. We can consider properties $\Gamma_{a}, \Gamma_{b}$ as input ones, as well as $\Gamma_{c}$ - as output properties. Therefore we can consider molecules $a$ and $b$ as input heterogeneous "signals", and molecule $c$ - as an output "signal" [17-19].

Formal approaches (10) and (11) allow one to develop an extended theory of heterogeneous automata including a theory of self-synchronized heterogeneous automata with individual names of transformed objects - "signals" [19].

Within the framework of GFT we are able to introduce a notion of so called "complete technology" T. Any technology $\mathbf{T}$ is called "complete" if it allows us: i) to obtain infinitely many new objects in the $\mathbf{T}$; ii) for any object (construction) made in the $\mathbf{T}$ we can restore its synthesis and structure even we get the object for the first time.

This notion is very important for the Formal Technology, because if technology $\mathbf{T}$ is complete, it means that we can use all possibilities of the $\mathbf{T}$. There are many complete technologies in GFT instead of one complete technology in computational mathematics called $\mu$-recursive or partial recursive functions (PRF). The completeness of PRF is achieved due to the equality predicate used in the minimization operator $\mu$ in PRF. In GFT the completeness of some technologies is achieved by unspecified analytical operations, i.e. by operations with unknown internal mechanisms and functional algorithms. Such unspecified analytical operations approximate Turing concepts of oracles 
introduced by Turing himself in his examples of some algorithmic problems [20]. Therefore various cognitive algorithms in GFT using such unspecified analytical operations (that acquire correct knowledge about the outer world) can be seen as examples of such algorithms with oracles [19].

On the basis of similar definition we are able to introduce a notion of "evolutional technology". A technology $\mathbf{T}$ is called evolutional if it allows us: i) to obtain infinitely many new objects (constructions) in the $\mathbf{T}$; ii) to define the novelty of every such new object (construction) exclusively by means of the same technology $\mathbf{T}$ (i.e. without any external subjects such as any person, men, mankind, God, and so on). In accordance with this definition the technology $\mathbf{T}_{\mathbf{v N}}$ of von Neumann self-reproducing cellular automata should be called evolutional one. Actually, $\mathbf{T}_{\mathbf{v N}}$ is very close to this definition except one possibility: $\mathbf{T}_{\mathbf{v N}}$ does not provide for object movements in a physical space, while an existent biological evolution provides for these movements. More precise analysis of necessary conditions for a development of bio-like evolutional technologies is made in [19].

It is possible to construct for GFT, as well as for PRF, an automatic system similars to Turing Machine $[7,8,10,12,19,21]$. Such a formal model of universal programmable systems for syntheses and analyses of various objects (in proper technology $\mathbf{T}$ ) is very suitable to construct a so-called "cognizer" - a model of programmable automata that can acquire a correct knowledge about the outer world $[19,22]$.

\section{Conclusions}

General Formal Technology offers formalisms that help us to understand many important problems in modern mathematics concerning the surrounding world, including various "Nature's inventions", its "intellect", basic logics of developments, and so on $[7-10,12,19,21,22]$. It is explained by specific formal representations of GFT objects in proper "physical algorithms" describing the real physical processes. These formalisms are very close to the formal structures of thing's properties in the real world and simultaneously to the well-known basic mathematical structures such as Maltsev's algebraic systems. So, we are able to formulate the following thesis:

Thesis 1. All surrounding physical processes, which are understandable ones, as well as till incomprehensible physical processes, are algorithmic ones.

This thesis could not be proved by mathematical means, because we have no formal mathematical definition of the term "physical process", as well as we have no formal mathematical definition of the term "man's calculation" for Church-Turing Thesis.

Mathematics widely uses algorithmic concepts, processes and notions. Moreover, this concept is a very important object for basic mathematical investigations at all. Therefore Thesis 1 could explain the universality and very broad applicability of mathematics itself.

\section{REFERENCES}

[1] Aristotle, Metaphysics, translated by W. D. Ross, 2000. Online available from

http://www.classicallibrary.org/aristotle/metaphysics/book01 .htm

[2] A. A. Lovelace, Notes by the Translator, in Faster than Thought. A Symposium on Digital Computing Machines, B. V. Bowden (ed.), Pitman and Sons, Ltd., London, 1953, pp.362-408.

[3] B. Jones, General System Theory and Algorithm Theory, Int. J. General Systems, 9, 157-160, 1983.

[4] W. Fontana, L.W. Buss, The barrier of objects: From dynamical systems to bounded organizations, in Boundaries and Barriers, J. Casti and A. Karlqvist (eds.), Addison-Wesley, pp. 56-116, 1996.

[5] J. Schmidhuber, Algorithmic Theories of Everything, Technical Report IDSIA-20-00, Version 2.0; 20 Dec 2000. Online available from http:/www.idsia.ch/ juergen/

[6] M. Tegmark, Is the Universe Made of Math? [Excerpt], Scientific American, January 10, 2014. Online available from $\mathrm{http}: / / \mathrm{www}$. scientificamerican.com/article/is-the-universe-m ade-of-math-excerpt/

[7] S.M. Krylov, Formal technology and universal systems I, II, Cybernetics and Systems Analysis, New York: Springer, ISSN 1060-0396 (Print) 1573-8337 (Online), Vol.22, Part I, N 4, pp.512-518; Part II, N 5, pp.567-572, 1986.

[8] S.M. Krylov, Ontologija meta-nauki. Aksiomy, tehnologii, algorytmy, evoljutcija, In Russ., (Ontology of Meta-Science. Axioms, Technologies, Algorithms, Evolution), LAP LAMBERT Academic Publishing, Saarbrücken, Deutchland, 2012. (ISBN 978-3-659-27964-5)

[9] S.M. Krylov, Mathematical Foundation of Metaphysics, Vestn. Samar. Gos. Tekhn. Univ. Ser. Fiz.-Mat. Nauki, № 1(26), pp. 233-242, 2012.

[10] S.M. Krylov, Prospects of Metamathematical Structures in Science, Vestn. Samar. Gos. Tekhn. Univ. Ser. Fiz.-Mat. Nauki, № 2(31), pp. 101-110, 2013, Online available from Http://mi.mathnet.ru/eng/vsgtu1203

[11] A.I. Malcev, Algebraic Systems, Springer-Verlag, Berlin, New York, 1973

[12] S.M. Krylov, Multifunctional Remote Labs for Real Experiments, Engineering Processes and Manufacturing Methods, International Journal of On-line Engineering, Vol. 10, № 5, pp.29-35, 2014. Online available from http://dx.doi.org/10.3991/ijoe.v10i5.3726

[13] K. Gödel, Über formal unentscheidbare Sätze der Principia Mathematica und verwandter Systeme I, Monatsheft für Math. und Physik, 38, 173-198, 1931, (see also 'On Formally Undecidable Propositions of Principia Mathematica And Related Systems', Kurt Gödel. Translated by B. Meltzer. Introduction by R. B. Braithwaite. University of Edinburgh, January, 1962. Online available from http://jacqkrol.x10.mx/assets/articles/godel-1931.pdf)

[14] G.J. Chaitin, A Century of Controversy over the Foundations 
of Mathematics, in Finite versus Infinite, C. Calude and G. Paun (eds.), Springer-Verlag London, pp.75-100, 2000.

[15] G.J. Chaitin, Proving Darwin. Making Biology Mathematical, New-York: Pantheon Books, 2012.

[16] J. von Neumann, Theory of Self-Reproducing Automata, Edited and completed by A.W. Burks. University of Illinois Press, Urbana and London, 1966.

[17] E. N. Grebenschikov, S. M. Krylov, Ontologija proektirovanija geterogennyh elektronnyh system, in Russ. (Ontology of Heterogeneous Electronic Systems Designs), Ontology of designing, № 1, pp.65-71, 2012.

[18] E. N. Grebenschikov, S. M. Krylov, Systemnyi analiz geterogennyh slabostrukturirovannyh avtomatov. II. Perehod ot preobrazovanij "himicheskogo tipa" $\mathrm{k}$ logicheskim shemam i obratno, in Russ., (Heterogeneous Weekly-Structured Automata. II. Conversion from «Chemical-Like Transformations» to Logical Ones and Vice
Versa), Vestn. Samar. Gos. Tekhn. Univ. Ser. Tehnich. Nauki, № 2(34), pp. 6-12, 2012.

[19] S.M. Krylov, Metamaticheskie osnovy nauki buduschego, in Russ., (Metamathematical Foundations of Future Science), Samara: SamGTU, (ISBN 978-5-7964-1686-0), 2014. Online available from http://vt.samgtu.ru/images/pdf/krylov/metamath.pdf

[20] A.M. Turing, Systems of Logic Based on Ordinals, Proc. London Math. Soc. s2-45 (1): 161-228, 1939. (Online available from http://www.dcc.fc.up.pt/ acm/turing-phd.pdf)

[21] S.M. Krylov, Universal Programmable Completely Automated Factories-on-a-Chip, in Proceedings of the $9^{\text {th }}$ International Conference on the Commercialization of Micro and Nano Systems COMS2004. Aug.29 - Sept.2, 2004, Edmonton, Alberta, Canada, pp.269-273, 2004.

[22] S.M. Krylov, Formal Technology and Cognitive Processes, Int. Journal of General Systems, 24, pp.233-243, 1996. 